\title{
Phenotype Specific Association of the TGFBR3 Locus with Nonsyndromic Cryptorchidism
}

\author{
Julia S. Barthold, * Yanping Wang, Thomas F. Kolon, Claude Kollin, \\ Agneta Nordenskjöld, Alicia Olivant Fisher, T. Ernesto Figueroa, \\ Ahmad H. BaniHani, Jennifer A. Hagerty, Ricardo Gonzalez, $\dagger$ \\ Paul H. Noh, $\neq$ Rosetta M. Chiavacci, Kisha R. Harden, Debra J. Abrams, \\ Cecilia E. Kim, Abigail B. Mateson, Alan K. Robbins, Jin Li, \\ Robert E. Akins, Jr., Hakon Hakonarson and Marcella Devoto
}

From Nemours Biomedical Research (JSB, YW, AOF, ABM, AKR, REA) and Division of Urology, Alfred I. duPont Hospital for
Children (JSB, TEF, AHBH, JAH, RG, PHN), Wilmington, Delaware, Division of Urology (TFK), Center for Applied Genomics
(RMC, KRH, DJA, CEK, JL, HH) and Division of Genetics (HH, MD), Children's Hospital of Philadelphia and Department of
Pediatrics (HH, MD), and Department of Biostatistics and Epidemiology (MD), Perelman School of Medicine, University of
Pennsylvania, Philadelphia, Pennsylvania, Department of Women's and Children's Health, Karolinska Institutet, Stockholm,
Sweden (CK, AN), and Department of Molecular Medicine, Sapienza University, Rome, Italy (MD)

Purpose: Based on a genome-wide association study of testicular dysgenesis syndrome showing a possible association with TGFBR3, we analyzed data from a larger, phenotypically restricted cryptorchidism population for potential replication of this signal.

Materials and Methods: We excluded samples based on strict quality control criteria, leaving 844 cases and 2,718 controls of European ancestry that were analyzed in 2 separate groups based on genotyping platform (ie Illumina ${ }^{\circledR}$ HumanHap550, version 1 or 3, or Human610-Quad, version 1 BeadChip in group 1 and Human OmniExpress 12, version 1 BeadChip platform in group 2). Analyses included genotype imputation at the TGFBR3 locus, association analysis of imputed data with correction for population substructure, subsequent meta-analysis of data for groups 1 and 2, and selective genotyping of independent cases (330) and controls (324) for replication. We also measured Tgfbr3 mRNA levels and performed TGFBR3/betaglycan immunostaining in rat fetal gubernaculum.

Results: We identified suggestive $\left(\mathrm{p} \leq 1 \times 10^{-4}\right)$ association of markers in/near TGFBR3, including rs9661103 (OR 1.40; 95\% CI 1.20, 1.64; $\mathrm{p}=2.71 \times 10^{-5}$ ) and rs10782968 (OR 1.58; 95\% CI 1.26, 1.98; $\mathrm{p}=9.36 \times 10^{-5}$ ) in groups 1 and 2 , respectively. In subgroup analyses we observed strongest association of rs17576372 (OR 1.42; 95\% CI 1.24, 1.60; p $=1.67 \times 10^{-4}$ ) with proximal and rs11165059 (OR 1.32; 95\% CI 1.15, 1.38; $\mathrm{p}=9.42 \times 10^{-4}$ ) with distal testis position, signals in strong linkage disequilibrium with rs9661103 and rs10782968, respectively. Association of the prior genome-wide association study signal ( $r$ 12082710) was marginal (OR 1.13; 95\% CI 0.99, 1.28; $\mathrm{p}=0.09$ for group 1), and we were unable to replicate signals in our independent cohort. Tgfbr3/betaglycan was differentially expressed in wild-type and cryptorchid rat fetal gubernaculum.

Conclusions: These data suggest complex or phenotype specific association of cryptorchidism with TGFBR3 and the gubernaculum as a potential target of TGF $\beta$ signaling.

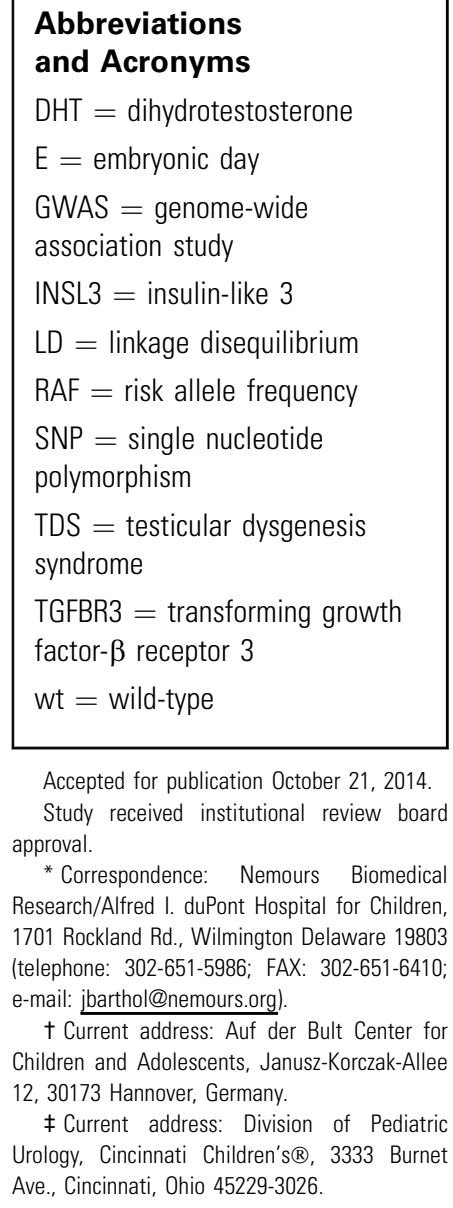

Accepted for publication October 21, 2014 Study received institutional review board approval.

* Correspondence: Nemours Biomedical Research/Alfred I. duPont Hospital for Children, 1701 Rockland Rd., Wilmington Delaware 19803 (telephone: 302-651-5986; FAX: 302-651-6410; e-mail: jbarthol@nemours.org).

† Current address: Auf der Bult Center for Children and Adolescents, Janusz-Korczak-Allee 12, 30173 Hannover, Germany.

₹ Current address: Division of Pediatric Urology, Cincinnati Children's ${ }^{\circledR}, 3333$ Burnet Ave., Cincinnati, Ohio 45229-3026. 
Key Words: cryptorchidism, genetic association studies, phenotype, testis

CRYPTORCHIDISM is a common genital anomaly identified in boys at birth or during childhood. The etiology is poorly understood and likely multifactorial, although associated genetic loci remain largely unknown. Familial aggregation suggests moderate genetic susceptibility and implicates the maternal environment as contributory to cryptorchidism risk. ${ }^{1,2}$ Level of familial risk in this disorder exceeds that for many complex diseases and predicts the potential for greater success in genomewide analyses. ${ }^{3}$

Testicular descent is regulated by insulin-like 3 and androgens, Leydig cell derived hormones that control development of the fetal gubernaculum. ${ }^{4}$ Exonic variants of INSL3, its receptor relaxin/insulin-like family peptide receptor 2 (RXFP2) or other hormone pathway genes are rare in cryptorchidism cases, and their functional significance is poorly defined. ${ }^{5}$ Dalgaard et al performed a GWAS augmented by systems biology analysis methodology to identify genetic markers linked to the testicular dysgenesis syndrome, which includes cryptorchidism, hypospadias, testicular cancer and infertility. ${ }^{6}$ This approach did not identify genome-wide significant signals, but had limited power to detect loci associated with isolated cryptorchidism. However, a TGFBR3 intronic single nucleotide polymorphism, rs12082710, demonstrated evidence suggestive of association with TDS and cryptorchidism. Accordingly we focused on TGFBR3 in an initial analysis of data from a larger GWAS cohort, and observed suggestive, phenotypespecific association of nonsyndromic cryptorchidism with this locus.

\section{MATERIAL AND METHODS}

\section{Subjects and Genotyping}

Subjects included boys with cryptorchidism who underwent surgical repair at Nemours/Alfred I. duPont Hospital for Children or the Children's Hospital of Philadelphia. Exclusion criteria consisted of multiple congenital anomalies and/or diagnosis of a syndrome, other genital anomalies (hypospadias, chordee or other penile anomalies) and abdominal wall defects or major urogenital malformations. Control subjects recruited through the Children's Hospital of Philadelphia Care Network were males 7 years or older without a history of testicular disease, syndromes or other medical disorders potentially associated with cryptorchidism, including inguinal hernia and hypospadias.

Basic demographic and phenotypic data were collected, including age at diagnosis, race, ethnicity, laterality and position of the affected testis. Blood sample or excess tissue was collected and stored at $-80 \mathrm{C}$ or in RNAlater ${ }^{\circledR}$ solution. As described previously, ${ }^{7}$ we categorized the cases into different phenotypic subgroups. Nonscrotal position was defined as distal if the most severely affected cryptorchid testis was located at or beyond the external inguinal ring and proximal if at least 1 testis was located within the inguinal canal or abdomen. We assigned boys 2 years or younger to the early subgroup and those older than 2 years to the late subgroup based on timing of surgery by a pediatric urologist. Informed consent was obtained for all participants based on approval of the institutional review board at each participating center.

DNA was extracted from tissue or blood samples (5 PRIME, Inc., Gaithersburg, Maryland), and whole genome amplification (REPLI-g Mini Kit, Qiagen, Germantown, Maryland) was performed for those with low DNA yield. Samples of adequate purity (optical density $260 / 280$ ratio of 1.8 to 2.0 by NanoDrop ${ }^{\circledR} 1000$ spectrophotometer) were entered into the standard genotyping work flow at the Center for Applied Genomics at the Children's Hospital of Philadelphia. Two separate groups were analyzed based on the genotyping platform used in the discovery stage, according to availability of control genotypes. Group 1 consisted of 559 cases and 1,772 controls that were genotyped using HumanHap550, version 1 or 3, or Human610-Quad, version 1 BeadChip (Illumina). These platforms have more than 535,000 SNPs in common. Group 2 consisted of 353 cases and 1,149 controls that were genotyped using the Human OmniExpress 12, version 1 BeadChip platform (Illumina).

\section{Discovery Phase Data Analysis}

Genome-wide genotyping data from groups 1 and 2 were analyzed separately using PLINK, version 1.07 (http:// pngu.mgh.harvard.edu/ purcell/plink/). ${ }^{8,9}$ SNP content differed slightly for each of the 3 genotyping platforms used in group 1. Therefore, only overlapping SNPs $(535,752)$ were used for subsequent analysis. Individuals were excluded from further analysis due to 1) discordance between reported sex and $\mathrm{X}$ and $\mathrm{Y}$ chromosome SNP data, 2) missing genotype rate greater than $3 \%, 3$ ) higher or lower than expected heterozygosity rate (greater than 3 SDs from mean) and 4) duplicates or relatives (based on estimate of proportion of alleles shared identical by descent greater than 0.1875). SNPs were excluded due to 1) missing genotype rate greater than $5 \%, 2$ ) HardyWeinberg equilibrium deviation in controls ( $\mathrm{p}<0.00001)$, 3) significantly different missing genotype rates between cases and controls ( $p<0.00001)$, and 4 ) low minor allele frequency (less than 0.01). To select samples of European ancestry and control for population substructure, multidimensional scaling analysis was performed in PLINK using European population SNP genotyping data from the Stanford Human Genome Diversity Project (http:// www.hagsc.org/hgdp/files.html). ${ }^{10}$ We removed all samples that deviated from the means of the first or second components by more than $3 \mathrm{SDs}$. We performed separate association analyses for the remaining samples in groups 
1 and 2 using logistic regression with multidimensional scaling components 1 and 2 as covariates.

We used HaploView, version 4.2 to define the pattern of LD at the TGFBR3 locus (http://www.broadinstitute.org/ scientific-community/science/programs/medical-andpopulation-genetics/haploview/haploview), ${ }^{11}$ and a targeted region was chosen for imputation (Chr1:9196000092415000, GRCh37/hg19 assembly). Genotype data in PLINK format were converted to IMPUTE, version 2.3.0 file format, ${ }^{12,13}$ using GTOOL, version 0.7.5 (http://www. well.ox.ac.uk/ cfreeman/software/gwas/gtool.html). We performed imputation using the 1000 Genomes Project reference population (September 2013 version), followed by association analysis of imputed data using IMPUTE2 and SNPTEST, version $2.5 \beta$ (https://mathgen.stats.ox.ac. uk/genetics_software/snptest/old/snptest_v2.3.0.html), respectively. ${ }^{12,13}$ Imputed SNPs were removed from SNPTEST results based on the criteria minor allele frequency less than 0.01, control Hardy-Weinberg equilibrium less than 0.00001 and frequentist_add_info (imputation quality score) less than 0.8. Meta-analyses of the remaining SNPs for groups 1 and 2 was performed using META, version 1.5 (http://www.stats.ox.ac.uk/ $\sim$ jsliu/meta.html) for all cases and controls, and for subgroups defined by testicular position, laterality and timing of presentation. Differences in phenotype frequencies between the 2 discovery groups were analyzed using chi-square tests. Results were mapped using the SNAP Web tool (http://www.broadinstitute.org/mpg/snap/), ${ }^{14}$ and LD among the strongest signals was estimated using HaploView.

\section{Replication Phase}

DNA samples from boys with cryptorchidism and unaffected controls were obtained in a prior study of cryptorchidism in Sweden. ${ }^{15-17}$ We selected the top TGFBR3 signals from groups 1 and 2, or an SNP in complete LD, and the previously published marker rs12082710 for genotyping using TaqMan ${ }^{\circledR}$ assays. ${ }^{6}$ Genotyping was performed in a 384-well format using the Applied Biosystems ${ }^{\circledR}$ PRISM ${ }^{\circledR} 7900 \mathrm{HT}$ fast real-time polymerase chain reaction system. A reaction volume of $5 \mu$ included $1.25 \mu \mathrm{l}$ water, $2.5 \mu \mathrm{l} 2 \times$ Applied Biosystems genotyping master mix, $0.25 \mu \mathrm{l} 20 \times$ TaqMan genotyping assay and $1 \mu \mathrm{l}$ (15 ng) genomic DNA. The polymerase chain reaction conditions were $95 \mathrm{C}$ for 10 minutes, followed by 50 cycles at $95 \mathrm{C}$ for 15 seconds and $60 \mathrm{C}$ for 60 seconds. The genotyping calls were made by Applied Biosystems SDS Automation Controller software, version 2.3. Association tests were performed using online software (http://ihg.gsf. de/cgi-bin/hw/hwa1.pl). A description of supplementary methods used to conduct real-time quantitative polymerase chain reaction and immunostaining is available online (http://jurology.com/). ${ }^{18-20}$

\section{RESULTS}

Based on quality control criteria, 56 of 559 cases, 158 of 1,772 controls and 28,749 of 535,752 markers were removed from group 1 , and 12 of 353 cases, 45 of 1,149 controls and 89,489 of 719,629 markers were removed from group 2 , leaving 844 cases and
2,718 controls in the final analysis. Q-Q plots of the distribution of $p$ values in groups 1 and 2 did not exhibit significant deviation from their expected distribution, with genomic inflation factor $(\lambda)$ values of 1.014 and 1.017 , respectively.

There were no genome-wide significant $\left(\mathrm{p}<5.0 \times 10^{-8}\right)$ signals in analyses of the 2 subsets in the discovery phase (groups 1 and 2), but we identified TGFBR3 (chr1:92145900-92371559, reverse strand, GRCh37/hg19 assembly) as the only genomic region containing suggestive $\left(p \leq 1 \times 10^{-4}\right)$ markers for both groups. In light of the previous data suggesting a possible association of TGFBR3 with TDS and cryptorchidism, ${ }^{6}$ we focused on this region initially. Following imputation the strongest signals were $\mathrm{rs} 9661103\left(\mathrm{p}=2.71 \times 10^{-5}\right)$, located in intron 1 , and $\mathrm{rs} 10782968\left(\mathrm{p}=9.38 \times 10^{-5}\right)$, located approximately $101 \mathrm{~kb}$ downstream between $T G F B R 3$ and $C D C 7$, for groups 1 and 2 , respectively (fig. 1, table 1). However, the reciprocal analysis revealed no evidence of association of $3^{\prime}$ downstream or $5^{\prime}$ intragenic SNPs with groups 1 and 2, respectively, so these signals were weaker when we combined discovery data for groups 1 and 2 in a meta-analysis. In addition, we observed marginal (OR $1.13,95 \%$ CI $0.99-1.28 \mathrm{p}=0.09$ ) association of rs12082710 with group 1 but not group 2 cases. However, the RAF in our control samples (0.62) was higher than previously reported (0.58 to 0.59$)$ for this variant, ${ }^{6}$ and, therefore, was similar to the RAF in our case groups. In the replication phase available genotypes of 305 cases and 324 unaffected controls collected from white subjects in Sweden failed to show association of any of these markers with cryptorchidism (table 1).

We performed additional analyses to determine whether phenotypic heterogeneity between our 2 case groups could potentially account for these variable results. Testicular position, laterality and/or age at presentation data were available for at least $90 \%$ of affected individuals in each classification (table 2). We noted increased frequencies of proximal, bilateral and early presentation in group 1 as compared to group 2, although these trends were not statistically significant. However, in metaanalyses of subphenotypes we noted strongest evidence of association of proximal and distal testicular positioning with upstream/promoter and downstream regions of TGFBR3, respectively (fig. 2, supplementary table [http://jurology.com/]). For the additional meta-analyses based on laterality and age at presentation all associations were weaker.

Using HaploView, we reviewed the LD patterns for the top signals in the 2 genotyping groups and the position subgroups. The strongest signals in group 1 (rs9661103) and group 2 (rs10782968) analyses are not in LD with each other, but were 

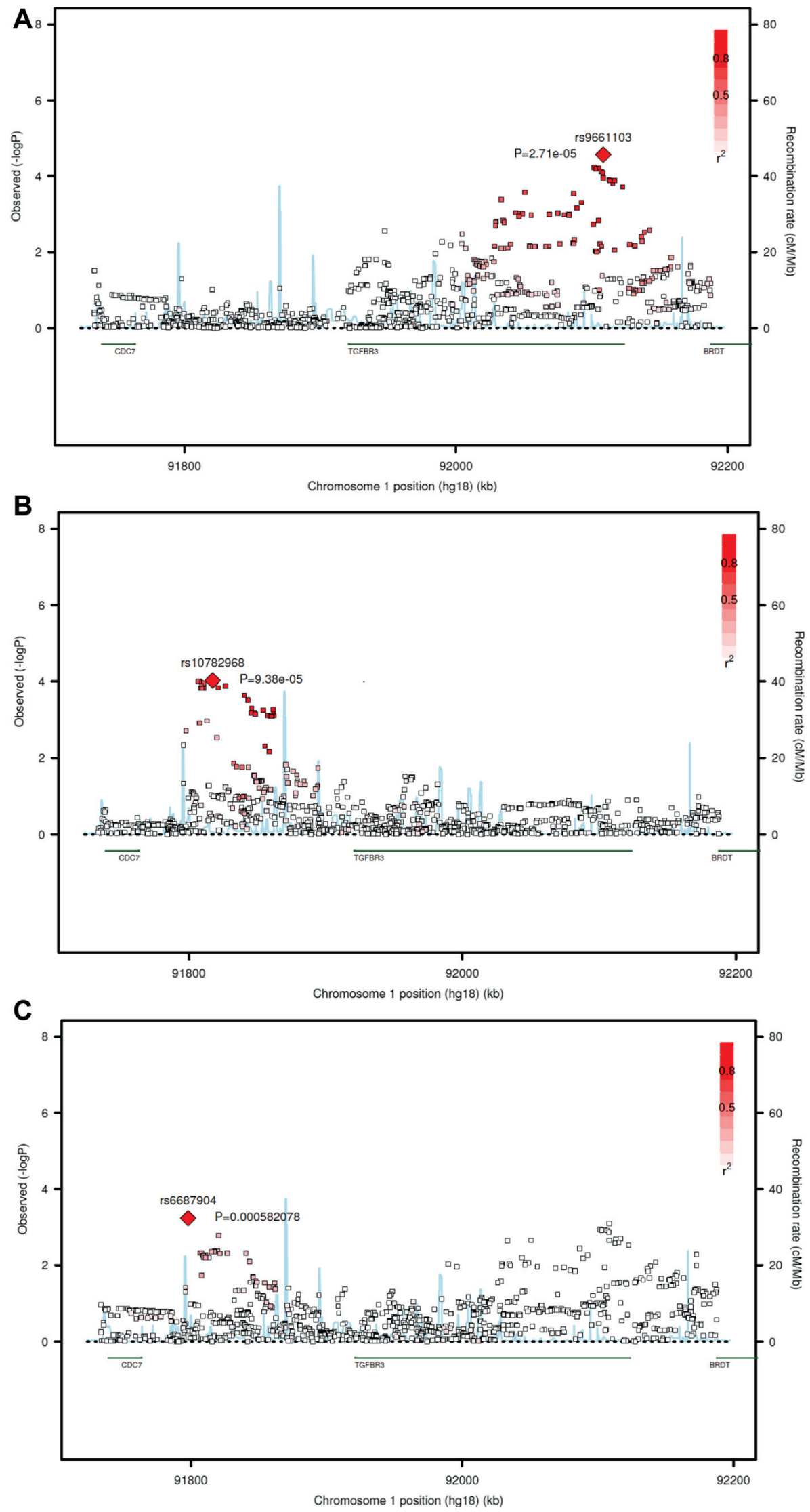

Figure 1. SNAP plots demonstrate association analysis results of imputed data. $A$, group 1 cases and controls. $B$, group 2 cases and controls. $C$, meta-analysis of all cases and controls. 
Table 1. Results of single marker association analysis in discovery and replication groups

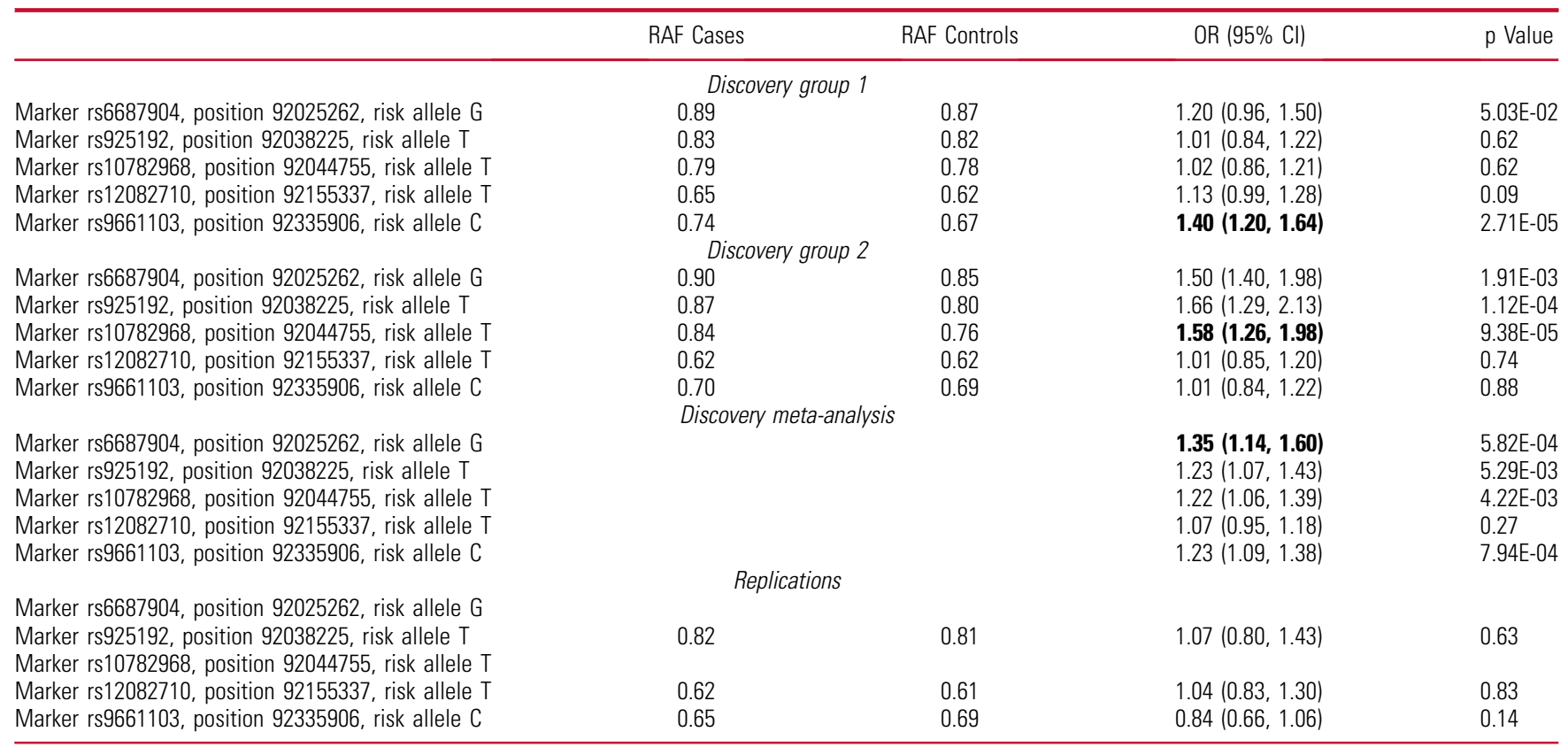

Markers shown include top signals (in bold) identified in groups 1 (rs9661103) and 2 (rs10782968 and rs925192) in present study, meta-analysis (rs6687904) and series by Dalgaard et al (rs12082710). ${ }^{6}$

correspondingly strongly linked $\left(r^{2} \geq 0.8\right)$ to the most significant SNPs that we identified in the proximal (rs17576372) and distal (rs6687904) meta-analyses (figs. 1 to 3 ), consistent with a greater frequency of proximal cases in group 1 . These results suggest that phenotypic bias may be 1 factor accounting for the nonoverlapping signals we identified in separate analyses of our discovery groups.

In studies of rat gubernaculum $T g f b r 3$ mRNA levels did not change significantly in wt samples between E17 and E21 ( $\mathrm{p}=0.57)$, the maximal developmental period (fig. 4). In contrast, Tgfbr3 expression increased after E17 $(\mathrm{p}=0.055)$ and was significantly greater in cryptorchid orl at E19 $(\mathrm{p}=0.011)$ and E21 $(\mathrm{p}=0.001)$ compared to wt gubernaculum. In wt gubernacular explants INSL3 exposure did not alter measured Tgfbr3 expression, but transcript levels increased significantly following DHT exposure ( $\mathrm{p}<0.01$ for $10 \mathrm{nM}$ DHT).

Table 2. Frequency of specific phenotypes by group

\begin{tabular}{llrr}
\hline \multicolumn{1}{c}{ Phenotype } & Group 1 & Group 2 & p Value \\
\hline $\begin{array}{l}\text { No. position (\%): } \\
\quad \text { Proximal } \\
\quad \text { Distal }\end{array}$ & $167(35)$ & $107(32)$ & 0.47 \\
$\begin{array}{l}\text { No. laterality (\%): } \\
\quad \text { Bilat }\end{array}$ & $313(65)$ & $224(68)$ & \\
$\quad$ Unilat & $137(28)$ & $80(24)$ & 0.21 \\
No. presentation (\%): & $360(72)$ & $258(76)$ & \\
$\quad$ Early & $236(48)$ & $142(42)$ & 0.07 \\
Late & $255(52)$ & $198(58)$ & \\
\hline
\end{tabular}

Immunostaining of the rat fetal gubernaculum demonstrated diffuse TGFBR3 protein expression within the inner mesenchymal core but more intense staining of the developing peripheral muscle and outer mesothelial layer, as noted in E17 and E19 wt gubernacula (fig. 5, A). The muscle layer was adjacent to the mesothelial layer at E17 and became progressively less peripheral and thicker during development. Prominent clusters of betaglycan (TGFBR3-A positive) immunoreactivity consistent with the proteolytically cleaved extracellular domain (soluble betaglycan) revealed variable expression at all time points, along with coexpression of membrane associated betaglycan and myosin in differentiated muscle. These extracellular collections were not identified using TGFBR3-B, an antibody targeting cytoplasmic betaglycan, and expression was less prominent by E21 in wt and orl samples. These collections were particularly prominent in some orl samples, particularly within the mesenchymal core and the peripheral mesothelial layer (fig. 5, B).

\section{DISCUSSION}

Cryptorchidism is the most common reproductive anomaly identified in newborn boys but genetic susceptibility loci remain largely undefined. In the sole published GWAS that includes cryptorchidism TGFBR3 was identified as a locus associated with TDS and less strongly with isolated nonsyndromic cryptorchidism, although the data did not approach 

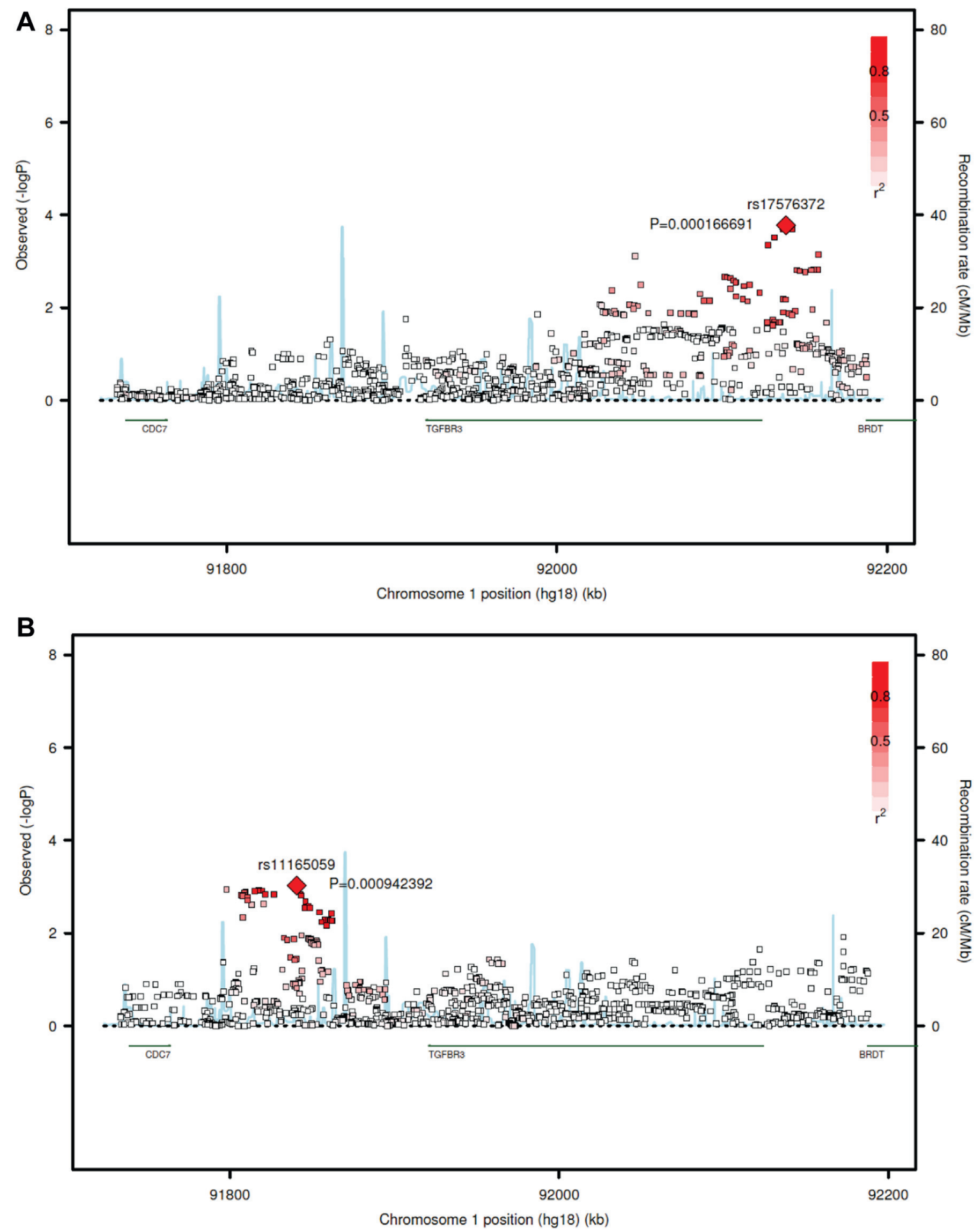

Figure 2. SNAP plots of meta-analysis results of imputed data at TGFBR3 locus. A, cryptorchid cases with testes located proximal to external inguinal ring and all controls. $B$, cryptorchid cases with testes located distal to external inguinal ring.

genome-wide significance. ${ }^{6}$ In the present analysis the larger sample size provided increased power to detect an association of TGFBR3 with cryptorchidism. Although we showed nominal association of our group 1 cohort with the previously reported signal, this association was lost with increasing sample size and was not replicated in a Swedish population. Surprisingly we observed suggestive $\left(\mathrm{p}<10^{-4}\right)$ association of each of our case-control groups with independent signals at or near the 


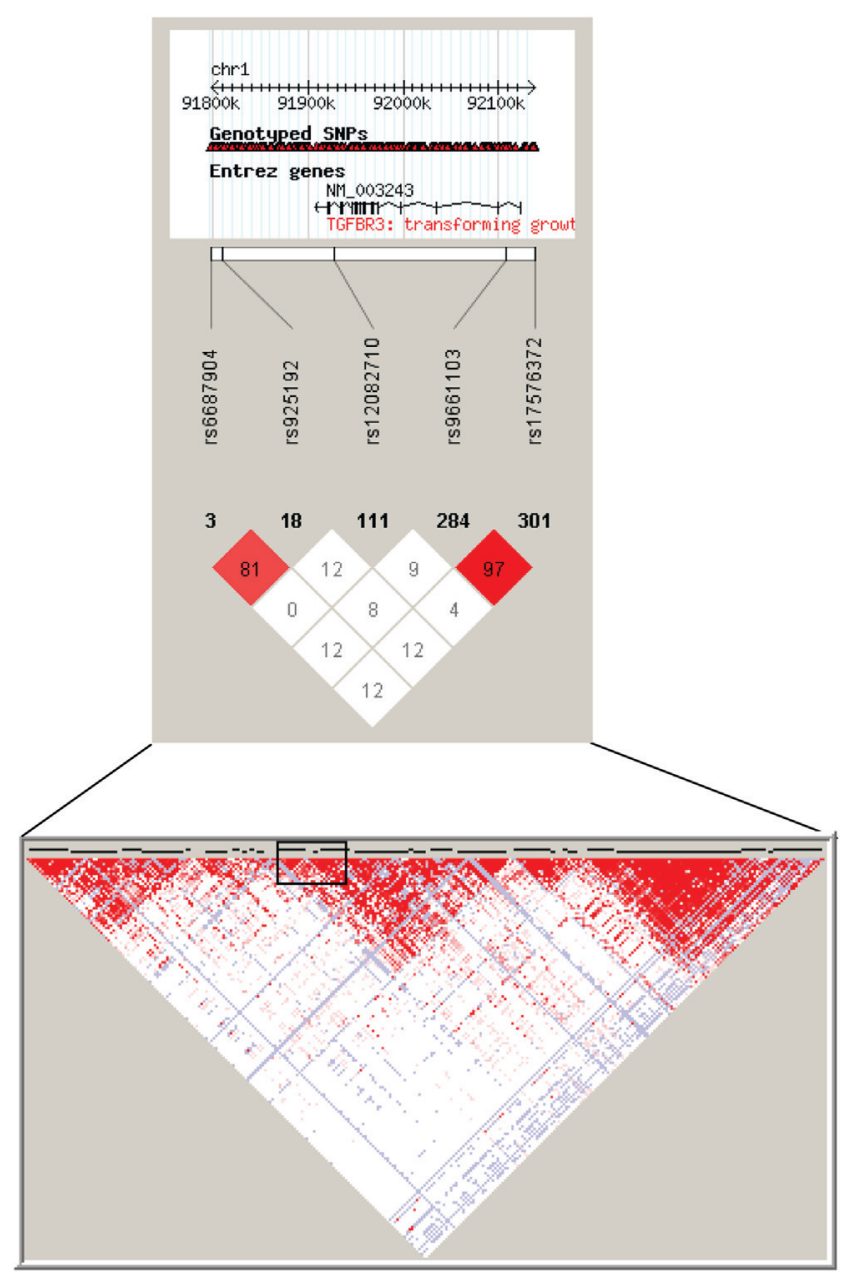

Figure 3. LD plot of markers identified in primary group and subgroup analyses (available in HaploView). Lower portion of figure illustrates overview of LD blocks within imputed TGFBR3 locus. Black rectangle is centered on $3^{\prime}$ end of TGFBR3 gene.

TGFBR3 locus. Our subphenotype analyses suggest stronger association of proximal and distal phenotypes with linked SNPs in the $5^{\prime}$ and downstream

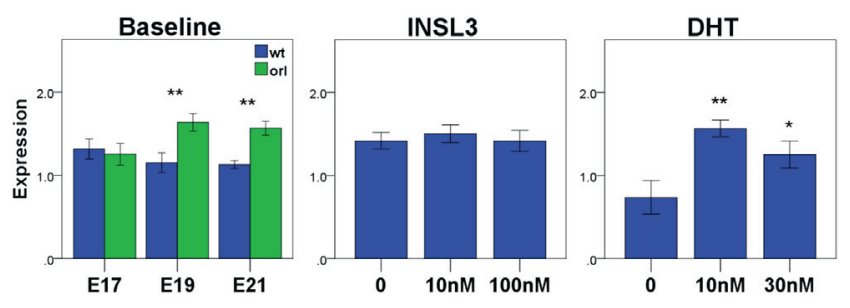

Figure 4. Tgfbr3 mRNA expression in gubernaculum from wt and cryptorchid orl rat fetuses. Baseline expression is illustrated for freshly isolated E17, E19 and E21 gubernacula and for E17 wt gubernacular explants established in culture followed by exposure to INSL3 or DHT for 24 hours. Asterisk indicates $p<0.05$ for wt vs orl. Double asterisk signifies $\mathrm{p}<0.01$ for wt vs orl.
A

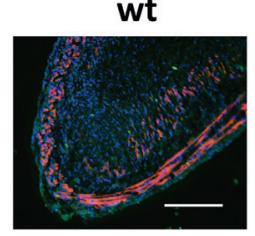

B

E17
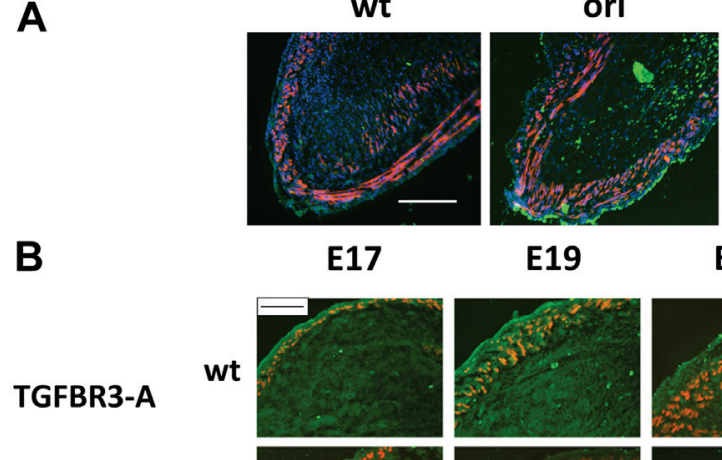

E19

E21

TGFBR3-B
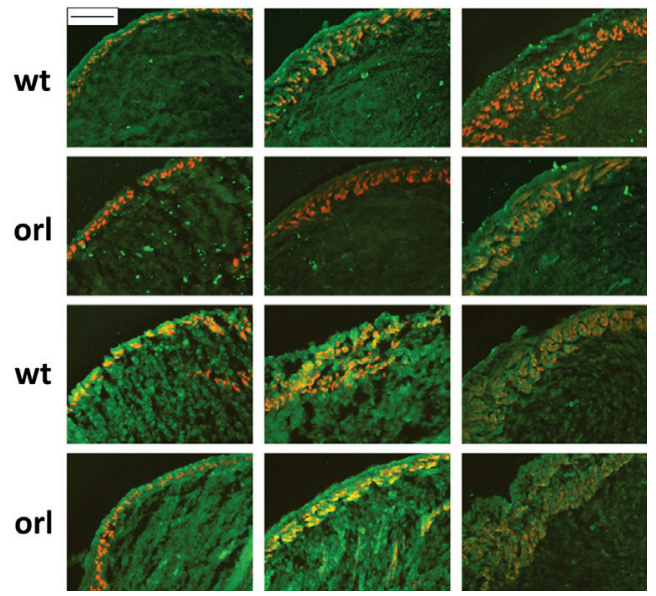

Figure 5. $A$, betaglycan expression (green stain) in E17, E19 and E21 gubernacula from wt and cryptorchid orl fetuses. Immunostaining reveals myosin (differentiated muscle, red) and TGFBR3-A (extracellular domain, green, top 2 rows) or TGFBR3-B (cystoplasmic domain, green, bottom 2 rows). Reduced from $\times 20$ (scale bar $100 \mu \mathrm{m}$ ). $B$, immunostaining for TGFBR3-A (green, 1:50), myosin (red) and nuclei (DAPI, blue) in E21 gubernaculum shows prominent soluble betaglycan collections in some fetuses. Reduced from $\times 10$ (scale bar $200 \mu \mathrm{m}$ ).

regions of the gene, respectively. HapMap data (http://hapmap.ncbi.nlm.nih.gov/cgi-perl/gbrowse/ hapmap24_B36/) demonstrate minimal LD between these 3 genomic regions. It is possible that the strength of the observed association for any 1 signal is limited by allelic heterogeneity and/or the unmeasured effects of variable environmental exposures.

Cryptorchidism is a complex disease, likely influenced by multilocus genetic, maternal and/or environmental factors. The genomic loci and pathways involved remain largely unknown, although many studies address this question in animal models. Gene targeting experiments in mice confirm requirement for $R x f p 2$ and $A r$, substantiate a role for Wnt and Notch signaling in gubernacular development, and confirm myogenesis as a key target of hormone action. ${ }^{21,22}$

TGFBR3/betaglycan is a biologically compelling risk locus for cryptorchidism because of its association with fetal Leydig cell development and myogenesis. ${ }^{23,24}$ Betaglycan is a TGF $\beta$ coreceptor that facilitates or inhibits signaling, depending on the context. ${ }^{25}$ The cytoplasmic domain facilitates 
signaling by TGF $\beta 2$ or TGF $\beta 1$, while the soluble, cleaved ectodomain can modulate TGF $\beta$ signaling by sequestering these growth factors. Betaglycan is expressed in interstitial and peritubular myoid cells in the fetal testes of mice and humans. ${ }^{26,27}$ Tgfbr3 - / - mice exhibit disruption of tubular architecture and a transient delay in Leydig cell development, with reduced expression of Insl3 and steroidogenesis genes. ${ }^{24}$ It is noteworthy that inactivation of $T g f \beta 2$ in transgenic mice is associated with cryptorchidism without other external genital defects, ${ }^{28}$ suggesting a primary gubernacular defect rather than global hormone deficiency. This and previous studies revealing expression of $\mathrm{Tgfbr} 3$ and $T g f b 2$ with increased expression in orl and wt males following androgen exposure support a role for TGF $\beta$ signaling in gubernaculum development. ${ }^{18,20}$

The present data suggest that membrane associated betaglycan is expressed throughout the fetal rat gubernaculum but most strongly in the outer mesothelial layer and in association with areas of developing muscle, and that focal increased accumulation of soluble betaglycan occurs in mesothelium and mesenchyme of orl gubernacula. Although we have not fully elucidated the genomic loci that confer susceptibility to cryptorchidism, our prior studies suggest that androgen signaling and muscle patterning are altered in the orl strain..$^{20,29}$
Inconsistency in the pattern of association of TGFBR3 with cryptorchidism may reflect various factors, notably insufficient sample size, allelic heterogeneity, population differences and/or phenotypic misclassification or variability. Regarding the latter, Dalgaard et al included subjects identified at birth or through medical records, ${ }^{6}$ although it is unclear whether all had persistent cryptorchidism requiring surgery. In their discussion of the concept of "synthetic" association of GWAS signals with rare, causal variants in the same genomic region Dickson et al also hypothesized that even modest associations defined by GWAS may show loci that contain rare variants of larger effect. ${ }^{30}$

\section{CONCLUSIONS}

The present data warrant more intensive investigation to define potential causal variants and the role of betaglycan in reproductive development in experimental animal models. Hypotheses that should be addressed include the possibility that genomic loci participate in hormone signaling pathways that are also specific targets of environmental exposures, and that pleiotropic effects of genes may explain the co-occurrence of testicular germ cell defects (cancer and subfertility) and cryptorchidism.

\section{REFERENCES}

1. Schnack TH, Zdravkovic S, Myrup C et al: Familial aggregation of cryptorchidism-a nationwide cohort study. Am J Epidemiol 2008; 167: 1453

2. Jensen MS, Toft G, Thulstrup AM et al: Cryptorchidism concordance in monozygotic and dizygotic twin brothers, full brothers, and halfbrothers. Fertil Steril 2010; 93: 124.

3. Hemminki K, Li $\mathrm{X}$, Sundquist $\mathrm{K}$ et al: Familial risks for common diseases: etiologic clues and guidance to gene identification. Mutat Res 2008; 658: 247.

4. Virtanen HE and Toppari J: Embryology and physiology of testicular development and descent. Pediatr Endocrinol Rev, suppl., 2014; 11: 206.

5. Ferlin A, Zuccarello D, Zuccarello B et al: Genetic alterations associated with cryptorchidism. JAMA 2008; 300: 2271.

6. Dalgaard MD, Weinhold N, Edsgärd D et al: A genome-wide association study of men with symptoms of testicular dysgenesis syndrome and its network biology interpretation. J Med Genet 2012; 49: 58.

7. Barthold JS, Hossain J, Olivant-Fisher A et al: Altered infant feeding patterns in boys with acquired nonsyndromic cryptorchidism. Birth Defects Res A Clin Mol Teratol 2012; 94: 900.

8. Anderson CA, Pettersson FH, Clarke GM et al: Data quality control in genetic case-control association studies. Nat Protoc 2010; 5: 1564.

9. Purcell S, Neale B, Todd-Brown K et al: PLINK a tool set for whole-genome association and population-based linkage analyses. Am J Hum Genet 2007; 81: 559.

10. Rosenberg NA: Standardized subsets of the HGDP-CEPH Human Genome Diversity Cell Line Panel, accounting for atypical and duplicated samples and pairs of close relatives. Ann Hum Genet 2006; 70: 841.

11. Barrett JC, Fry B, Maller J et al: Haploview: analysis and visualization of $\mathrm{LD}$ and haplotype maps. Bioinformatics 2005; 21: 263.

12. Howie B, Marchini J and Stephens M: Genotype imputation with thousands of genomes. G3 (Bethesda) 2011; 1: 457.

13. Howie BN, Donnelly P and Marchini J: A flexible and accurate genotype imputation method for the next generation of genome-wide association studies. PLoS Genet 2009; 5: e1000529.
14. Johnson $A D$, Handsaker RE, Pulit SL et al: SNAP: a Web-based tool for identification and annotation of proxy SNPs using HapMap. Bioinformatics 2008; 24: 2938.

15. Kollin C, Hesser U, Ritzén EM et al: Testicular growth from birth to two years of age, and the effect of orchidopexy at age nine months: a randomized, controlled study. Acta Paediatr 2006; 95: 318.

16. Kollin C, Karpe B, Hesser U et al: Surgical treatment of unilaterally undescended testes: testicular growth after randomization to orchiopexy at age 9 months or 3 years. J Urol 2007; 178: 1589

17. Kollin C, Stukenborg JB, Nurmio M et al: Boys with undescended testes: endocrine, volumetric and morphometric studies on testicular function before and after orchidopexy at nine months or three years of age. J Clin Endocrinol Metab 2012; 97: 4588

18. Barthold JS, Wang Y, Robbins A et al: Transcriptome analysis of the dihydrotestosteroneexposed fetal rat gubernaculum identifies common androgen and insulin-like 3 targets. Bio Reprod 2013; 89: 143.

19. Johnson KJ, Robbins AK, Wang Y et al: Insulinlike 3 exposure of the fetal rat gubernaculum 
modulates expression of genes involved in neural pathways. Biol Reprod 2010; 83: 774.

20. Barthold JS, Robbins A, Wang $Y$ et al: Cryptorchidism in the orl rat is associated with muscle patterning defects in the fetal gubernaculum and altered hormonal signaling. Biol Reprod 2014; 91: 41.

21. Kaftanovskaya EM, Feng S, Huang $Z$ et al: Suppression of insulin-like3 receptor reveals the role of $\beta$-catenin and Notch signaling in gubernaculum development. Mol Endocrinol 2011; 25: 170.

22. Kaftanovskaya EM, Huang Z, Barbara AM et al: Cryptorchidism in mice with an androgen receptor ablation in gubernaculum testis. Mol Endocrinol 2012; 26: 598.

23. Lopez-Casillas F, Riquelme C, Perez-Kato $Y$ et al: Betaglycan expression is transcriptionally up-regulated during skeletal muscle differentiation. Cloning of murine betaglycan gene promoter and its modulation by MyoD, retinoic acid, and transforming growth factor-beta. J Biol Chem 2003; 278: 382

24. Sarraj MA, Escalona RM, Umbers A et al: Fetal testis dysgenesis and compromised Leydig cell function in Tgfbr3 (beta glycan) knockout mice. Biol Reprod 2010; 82: 153.

25. Bilandzic M and Stenvers KL: Betaglycan: a multifunctional accessory. Mol Cell Endocrinol 2011; 339: 180.

26. Anderson RA, Cambray N, Hartley PS et al: Expression and localization of inhibin alpha, inhibin/activin betaA and betaB and the activin type II and inhibin beta-glycan receptors in the developing human testis. Reproduction 2002; 123: 779 .
27. Sarraj MA, Chua HK, Umbers A et al: Differential expression of TGFBR3 (betaglycan) in mouse ovary and testis during gonadogenesis. Growth Factors 2007; 25: 334.

28. Sanford LP, Ormsby I, Gittenberger-de Groot AC et al: TGFbeta2 knockout mice have multiple developmental defects that are non-overlapping with other TGFbeta knockout phenotypes. Development 1997; 124: 2659.

29. Johnson KJ, McCahan SM, Si X et al: The orl rat with inherited cryptorchidism has increased susceptibility to the testicular effects of in utero dibutyl phthalate exposure. Toxicol Sci 2008; 105: 360 .

30. Dickson SP, Wang K, Krantz I et al: Rare variants create synthetic genome-wide associations. PLoS Biol 2010; 8: e1000294. 